\title{
Correction to: Identification Numbers and Modular Arithmetic
}

\section{Correction to:}

Chapter 1 in: D.R. Finston and P.J. Morandi, Abstract Algebra: Structure and Application, Springer Undergraduate Texts in Mathematics and Technology, https://doi.org/10.1007/978-3-319-04498-9_1

In the original version of Chapter 1, the figure was wrong which has now been replaced, at the end of the chapter opening page. 